\title{
Schemi di terapia immunosoppressiva e livelli ematici consigliati
}

\author{
M.P. Scolari, G. Comai, G. Liviano d'Arcangelo, P. Todeschini, G. Mosconi, \\ G. La Manna, G. Feliciangeli, S. Stefoni
}

U.O. di Nefrologia, Dialisi e Trapianto, Policlinico S. Orsola-Malpighi, Bologna

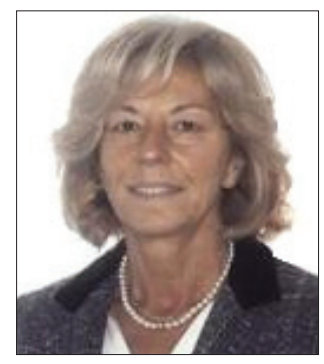

Maria Pia Scolari
Il percorso terapeutico del paziente trapiantato di rene comprende due periodi principali: il periodo iniziale in cui la terapia immunosoppressiva è massimale (di solito dalle prime 2-3 settimane fino ai primi 2-3 mesi post-trapianto) e il periodo di mantenimento, vale a dire tutto il periodo del follow-up del paziente dal momento in cui viene raggiunto lo steady state clinico/farmacologico. Sia nel primo periodo che nel mantenimento gli schemi di terapia prevedono generalmente la somministrazione di due o più farmaci immunosoppressori diversi per meccanismo d'azione ed effetti collaterali, con l'intento di potenziare l'efficacia immunosoppressiva e ridurre le complicanze legate al singolo farmaco. Tali farmaci possono rimanere gli stessi, con modificazioni solo posologiche nel corso degli anni, oppure possono essere variati in base alle esigenze cliniche del paziente e alle modificazioni funzionali del rene trapiantato (1).

Nel periodo iniziale, oltre alla terapia di base, nella maggior parte dei casi oggi viene effettuata la terapia di induzione, la cui somministrazione inizia nell'immediato pre-trapianto; tale terapia consta di farmaci non depletivi dei linfociti (anticorpi monoclonali) o farmaci depletivi dei linfociti (anticorpi mono o policlonali). La terapia di induzione con farmaci depletivi è fortemente consigliata nei pazienti iperimmunizzati o nei casi in cui sia prevista una prolungata sofferenza ischemica dell'organo (2).

In modo schematico la terapia immunosoppressiva può essere suddivisa come segue:

- terapia "standard": schemi di terapia di largo impiego e consolidati in base ai dati della letteratura;

- terapia "personalizzata": schemi adattati alle esigen- ze del "trapianto" (donatore non ottimale, ricevente iperimmunizzato, comorbidità nel ricevente ecc.), validati dai nuovi trial nazionali o internazionali oppure basati sull'esperienza dei singoli Centri Trapianto.

\section{Schemi di terapia "standard"}

I regimi immunosoppressivi standard consistono nella somministrazione di un solo farmaco, di solito un inibitore della calcineurina $(\mathrm{CNI})$, seppure nei pazienti trapiantati prima degli anni Ottanta è possibile riscontrare anche l'azatioprina, associata allo steroide (Schema Duplice); oppure di due immunosoppressori a differente meccanismo d'azione, di solito un CNI con un inibitore della sintesi del DNA o un inibitore della sintesi di mTOR, sempre in associazione con lo steroide (Schema Triplice). Nel corso degli anni si osserva che lo Schema Duplice è sempre meno utilizzato e viene riservato a quei soggetti che sviluppano "intolleranza" all'immunosoppressore di accompagnamento (3) (Tab. I).

TABELLA I - SCHEMI DI TERAPIA IMMUNOSOPPRESSIVA "STANDARD"

\begin{tabular}{|l|}
\hline SCHEMA DUPLICE \\
\hline Steroide + Ciclosporina \\
\hline Steroide + Tacrolimus \\
\hline Steroide + Azatioprina \\
\hline \\
\hline SCHEMA TRIPLICE \\
\hline Steroide + Ciclosporina + Azatioprina \\
\hline Steroide + Ciclosporina + Acido Micofenolico \\
\hline Steroide + Tacrolimus + Acido Micofenolico \\
\hline Steroide + Ciclosporina + Inibitore di mTOR \\
\hline Steroide + Tacrolimus + Inibitore di mTOR \\
\hline
\end{tabular}


Vengono illustrate di seguito le posologie medie dei farmaci nell'ambito delle terapie standard e le concentrazioni ematiche suggerite per alcuni di essi $(\mathrm{C} 0=$ concentrazione basale, al mattino immediatamente prima della assunzione del farmaco; $\mathrm{C} 2$ = concentrazione di picco, dopo 2 ore dalla assunzione del farmaco):

\section{1) SCHEMA DUPLICE}

Steroide: generalmente lo steroide viene prescritto per tutto il periodo di mantenimento al dosaggio di $2,5-5 \mathrm{mg} /$ die di prednisone o $2-4 \mathrm{mg} /$ die di metilprednisolone.

Ciclosporina (preparazione in microemulsione): viene mediamente prescritta a un dosaggio di $4-6 \mathrm{mg} / \mathrm{kg}$ in due somministrazioni per ottenere i seguenti livelli ematici:

- C0 250-550 ng/mL, C2 1000-1500 ng/mL per i primi 3 mesi

- C0 150-350 ng/mL, C2 700-1000 ng/mL successivamente.

Tacrolimus: viene mediamente prescritto a una posologia di $0,2 \mathrm{mg} / \mathrm{kg}$ in due somministrazioni per ottenere $\mathrm{i}$ seguenti livelli ematici basali:

- C0 $8-15 \mathrm{ng} / \mathrm{mL}$ per i primi tre mesi

- C0 6-10 ng/mL successivamente.

Azatioprina: viene prescritta alla dose iniziale di 1,5-2 $\mathrm{mg} / \mathrm{kg}$ e successivamente alla dose di $0,8-1 \mathrm{mg} / \mathrm{kg}$; tale farmaco oggi è sempre meno utilizzato, ad eccezione dei pazienti trapiantati da molti anni, per l'assenza di selettività nel meccanismo d'azione e per l'effetto mielotossico.

\section{2) SCHEMA TRIPLICE}

Steroide: generalmente lo steroide viene prescritto per tutto il periodo di mantenimento al dosaggio di 2,5-5 mg/ die di prednisone o $2-4 \mathrm{mg} /$ die di metilprednisolone.

Ciclosporina (preparazione in microemulsione): viene mediamente prescritta a un dosaggio di $4-6 \mathrm{mg} / \mathrm{kg}$ in due somministrazioni per ottenere i seguenti livelli ematici:

- C0 150-400 ng/mL, C2 800-1200 ng/mL per i primi 3 mesi

- C0 100-300 ng/mL, C2 700-900 ng/mL successivamente.

Tacrolimus: viene mediamente prescritto a una posologia di $0,2 \mathrm{mg} / \mathrm{kg}$ in due somministrazioni per ottenere i seguenti livelli ematici basali:

- C0 8-12 ng/mL per i primi tre mesi

- C0 5-8 ng/mL successivamente.
Azatioprina: viene prescritta alla dose di 0,8-1 mg/kg.

Farmaci a base di Acido Micofenolico (MMF= Micofenolato Mofetile o EC-MPS = Micofenolato Sodico gastroprotetto): tali farmaci vengono prescritti alla posologia di $2 \mathrm{gr} / \mathrm{die}$ o $1440 \mathrm{mg} /$ die inizialmente, $1 \mathrm{gr} /$ die o $720 \mathrm{mg} /$ die successivamente, in due somministrazioni.

Inibitori di mTOR: comprendono due farmaci attualmente in commercio registrati per la terapia immunosoppressiva che sono Sirolimus ed Everolimus.

- Sirolimus prescritto alla dose di carico di 4-6 mg in unica somministrazione il primo giorno e successivamente $2 \mathrm{mg} /$ die per mantenere livelli ematici di $8-15 \mathrm{ng} / \mathrm{mL}$ nei primi 3 mesi e $5-12 \mathrm{ng} / \mathrm{mL}$ successivamente.

- Everolimus prescritto alla dose iniziale di $1,5 \mathrm{mg} /$ die in due somministrazioni per mantenere livelli ematici di 8-12 ng/mL nei primi 3 mesi e 3-8 ng/mL successivamente.

Lo schema standard, in particolare lo schema Triplice, è stato fino ad oggi molto utilizzato nel trapianto di rene, in quanto ha permesso di ridurre drasticamente lincidenza del rigetto acuto e di aumentare la sopravvivenza dell'organo nel breve e nel medio termine. Tali spettacolari risultati non sono stati confermati nel lungo termine; inoltre nel corso degli ultimi anni si è osservato un importante cambiamento dello scenario trapiantologico con aumento del numero di donatori non ottimali e di riceventi gravati da numerose comorbidità. Per questo motivo sono stati introdotti nuovi schemi di terapia immunosoppressiva che tengono conto di tali problematiche (4).

\section{Schemi di terapia "personalizzati"}

Gli schemi di terapia personalizzati (5) possono essere suddivisi in:

1) schemi che prevedono l'assenza degli steroidi o la loro sospensione;

2) schemi che prevedono la minimizzazione degli inibitori della calcineurina;

3) schemi completamente privi degli inibitori della calcineurina.

1) Schemi di terapia che prevedono l'assenza degli steroidi o la loro sospensione

a) Schemi senza steroidi

I numerosi trial della letteratura che non prevedono lo 
steroide, evidenziano un'incidenza di rigetto acuto maggiore rispetto ai trattamenti che lo contemplano (6). Per tale motivo questi schemi di solito prevedono la contemporanea somministrazione della terapia di induzione con farmaci depletivi dei linfociti. Gli schemi senza steroide sono particolarmente indicati in tutte le categorie di pazienti a maggior rischio di sviluppo di complicanze steroido-relate (pazienti anziani, pazienti ad elevato rischio cardiovascolare, ad elevato rischio di sviluppare patologia ossea, cataratta, pazienti diabetici, obesi ecc.). Questi schemi sono al contrario controindicati nei pazienti ad elevato rischio immunologico o portatori di una nefropatia o una patologia di base che necessita di terapia steroidea continuativa.

Lo steroide viene effettuato solo il giorno del trapianto (perioperatorio) o, insieme al farmaco di induzione (per esempio, immunoglobulina anti-timocitica), per alcuni giorni.

Generalmente questi schemi prevedono un inibitore della calcineurina (ciclosporina o tacrolimus) associato ad acido micofenolico o ad inibitori di mTOR. La maggior parte degli studi utilizza Tacrolimus come inibitore della calcineurina. In generale non è consigliata la minimizzazione dei livelli ematici dell'inibitore della calcineurina. Per i livelli da mantenere si rimanda a quelli riportati negli schemi "standard".

$\grave{E}$ inoltre indicato un attento monitoraggio clinico laboratoristico per prevenire l'insorgenza di un'attivazione immunologica che, se presente, costringe in molti casi all'introduzione dello steroide.

\section{b) Schemi che sospendono lo steroide}

La sospensione può essere precoce cioè entro i primi tre mesi, oppure tardiva (oltre il $3^{\circ}$ mese). La sospensione precoce ha il vantaggio di poter verificare con sicurezza l'eventuale comparsa di una reazione immunologica; il paziente infatti è ancora sottoposto a controlli clinicolaboratoristici ravvicinati che permettono di evidenziare immediatamente anche un rigetto sub-clinico.

In letteratura la maggior parte degli studi prevede la sospensione degli steroidi tra il $3^{\circ}$ e il $6^{\circ}$ mese dal trapianto e tale modalità non è associata a un aumento della mortalità o della perdita del trapianto. Il rischio di rigetto acuto sembra essere maggiore se la terapia associata è la sola ciclosporina. Appare quindi indicata una terapia che preveda, almeno inizialmente, l'inibitore della calcineurina a dosaggio standard associato all'acido micofenolico o all'inibitore di mTOR (7).

Per i pazienti che effettuano terapia steroidea da molti anni, la sospensione dello steroide, pur essendo sempre possibile, va effettuata con estrema cautela e solo nei casi in cui vi siano conclamati effetti collaterali relati allo steroide; la sospensione infatti può alterare l'equilibrio immunologico o evidenziare più rapidamente la tendenza all'evoluzione verso l'insufficienza funzionale del rene trapiantato. La sospensione inoltre va effettuata molto lentamente per evitare una sindrome da insufficienza surrenalica.

\section{2) Schemi che prevedono la minimizzazione degli inibitori della calcineurina}

Il trattamento con inibitori della calcineurina si associa nel tempo al rischio di nefrotossicità, pertanto una strategia terapeutica di recente introduzione prevede la minimizzazione di tali farmaci in associazione ad acido micofenolico o ad inibitori di mTOR $(8,9)$ (Tab. II).

Questi schemi sono indicati per la maggior parte dei pazienti ma, soprattutto, in quei casi in cui siano ipotizzabili alterazioni parenchimali ischemiche o sclerotiche a carico del rene trapiantato; sono meno indicati nei pazienti iperimmunizzati per il maggior rischio di sviluppare un rigetto acuto.

E importante sottolineare che i dosaggi di questi farmaci sono solo indicativi e vanno adattati alle esigenze del singolo paziente. La risposta immunosoppressiva di ogni paziente è infatti diversa sia per i fattori genetici che regolano il metabolismo dei farmaci sia per la predisposizione alle infezioni o al rigetto. Sono schemi innovativi e necessitano di ulteriori conferme della loro efficacia e sicurezza nel lungo termine.

\section{3) Schemi di terapia privi degli inibitori della calcineurina}

Questi schemi sono di due tipi: schemi di conversione e schemi completamente privi di inibitori della calcineurina.

\section{a) Schemi di conversione}

Dopo un iniziale periodo di trattamento a base di CNI, questi vengono completamente sospesi e sostituiti.

Tale schema viene utilizzato nel trattamento di pazienti in terapia di base con CNI e con una diagnosi certa di disfunzione cronica da trapianto, dovuta prevalentemente alla nefrotossicità di tali farmaci in assenza di fattori immunologici, nel tentativo di rallentare il peggioramento funzionale.

La conversione viene generalmente effettuata sostituendo i CNI con gli mTOR in quanto farmaci meno nefrotossici e in grado di prevenire la proliferazione dell'endotelio vascolare sia sull'organo che sul paziente. I risultati della letteratura (peraltro gravati da numerose criticità 
TABELLA II - SCHEMI DI TERAPIA IMMUNOSOPPRESSIVA CON MINIMIZZAZIONE DI CNI

Ciclosporina 1,5-2 $\mathrm{mg} / \mathrm{kg}$ in due somministrazioni per ottenere i seguenti livelli ematici basali:

C0 150-250 ng/mL, C2 $600-900 \mathrm{ng} / \mathrm{mL}$ per i primi 3 mesi

C0 50-150 ng/mL, C2 250-450 ng/mL successivamente

Acido micofenolico

(MMF 2 gr/die o EC-MPS $1440 \mathrm{mg} / \mathrm{die}$ )

Tacrolimus $0,1 \mathrm{mg} / \mathrm{kg}$ in due somministrazioni per ottenere i seguenti livelli ematici basali:

C0 $5-8 \mathrm{ng} / \mathrm{mL}$ per i primi tre mesi

C0 $3-5 \mathrm{ng} / \mathrm{mL}$ successivamente

Acido micofenolico

(MMF $1 \mathrm{gr} /$ die o EC-MPS $720 \mathrm{mg} / \mathrm{die}$ )

Ciclosporina 1,5-2 mg/kg in due somministrazioni per ottenere i seguenti livelli ematici basali:

C0 $150-250 \mathrm{ng} / \mathrm{mL}, \mathrm{C} 2600-900 \mathrm{ng} / \mathrm{mL}$ per i primi 3 mesi

C0 50-150 ng/mL, C2 250-450 ng/mL successivamente

Sirolimus

C0 8-15 per i primi 3 mesi

C0 $4-8 \mathrm{ng} / \mathrm{mL}$ successivamente

Tacrolimus $0,1 \mathrm{mg} / \mathrm{kg}$ in due somministrazioni per ottenere i seguenti livelli ematici basali:

C0 $5-8 \mathrm{ng} / \mathrm{mL}$ per i primi tre mesi

C0 $3-5 \mathrm{ng} / \mathrm{mL}$ successivamente

Ciclosporina $1,5-2 \mathrm{mg} / \mathrm{kg}$ in due somministrazioni per ottenere i seguenti livelli ematici basali:

C0 150-250 ng/mL, C2 600-900 ng/mL per i primi 3 mesi

C0 50-150 ng/mL, C2 250-450 ng/mL successivamente

Sirolimus

C0 $8-15$ per i primi 3 mesi

C0 $4-8 \mathrm{ng} / \mathrm{mL}$ successivamente

\section{Everolimus}

C0 8-12 per i primi 3 mesi

C0 5-10 ng/mL successivamente

Tacrolimus $0,1 \mathrm{mg} / \mathrm{kg}$ in due somministrazioni per ottenere i seguenti livelli ematici basali:

C0 $5-8 \mathrm{ng} / \mathrm{mL}$ per i primi tre mesi

C0 $3-5 \mathrm{ng} / \mathrm{mL}$ successivamente

Everolimus

C0 8 -12 per i primi 3 mesi

C0 $5-10 \mathrm{ng} / \mathrm{mL}$ successivamente quali la disomogeneità della casistica, la variabilità del momento della conversione ed il breve follow-up) sono però spesso contradditori (10).

Le indicazioni alla conversione riguardano soprattutto quei casi in cui le lesioni istologiche siano ancora modeste, la funzione renale moderatamente compromessa $\left(\mathrm{GFR}>40 \mathrm{ml} / \mathrm{min} / 1,73 \mathrm{~m}^{2}\right)$ e non sia presente una proteinuria significativa (superiore a $800-1000 \mathrm{mg} / \mathrm{die}$ ). Gli inibitori di mTOR infatti caratteristicamente possono determinare l'insorgenza di proteinuria o aggravare la proteinuria già esistente con compromissione della funzionalità renale nel lungo termine (11).

I più moderni approcci terapeutici propongono tale schema in pazienti con trapianto normofunzionante al fine di prevenirne la disfunzione cronica nel lungo termine; in questi casi la conversione può essere effettuata precocemente (di solito entro il terzo mese) o in fasi successive.

Alla sospensione dei CNI, la terapia consiste nell'associazione di mTOR e acido micofenolico con o senza steroide. I livelli raccomandati di mTOR non differiscono da quelli degli schemi standard (sirolimus 5-10 ng/mL, everolimus 5-8 $\mathrm{ng} / \mathrm{mL}$ ) in associazione a MMF 1-2 gr/die o ECMPS 720-1440 mg/die. Tale schema necessita di un attento monitoraggio in particolare per il possibile sommarsi di effetti indesiderati secondari all'azione di entrambi i farmaci sul midollo osseo e sull'apparato gastro-intestinale (anemia, leucopenia, piastrinopenia, diarrea, aftosi ecc.) (12).

\section{b) Schemi completamente privi di inibitori della calcineurina}

L'approccio terapeutico che non prevede la somministrazione dell'inibitore della calcineurina fin dall'inizio è stato introdotto per cercare di preservare la funzione renale il più a lungo possibile $\mathrm{e}$ per una migliore conservazione del parenchima renale, evitando l'effetto nefrotossico legato ai CNI. L'utilizzo de novo del solo MMF in associazione allo steroide non ha dimostrato risultati soddisfacenti per l'eccessiva incidenza di episodi di rigetto acuto; schemi con mTOR inibitori associati ad acido micofenolico e steroidi sono oggi più utilizzati soprattutto in casi particolari (trapianto da donatore non ottimale). E consigliabile l'uso di una terapia di induzione (di solito con immunoglobuline anti-timociti) in quanto è segnalata un'incidenza di rigetto acuto più elevata rispetto ai trattamenti che prevedono l'uso di CNI. Inoltre, come specificato precedentemente, l'associazione di questi due farmaci può essere gravata da effetti collaterali importanti.

L'esperienza della letteratura è abbastanza incoraggiante riguardo questo approccio terapeutico, ma la maggior parte dei risultati riportati fanno riferimento solo al breve termine $(13,14)$. 


\section{Variazioni terapeutiche nel corso della vita del trapianto}

La perdita dell'organo trapiantato può avvenire per varie cause sia immunologiche che non immunologiche, ma in ultima analisi le più importanti sono le seguenti: rigetto cronico, fibrosi/atrofia parenchimale, morte del paziente con rene funzionante, per patologia infettiva, neoplastica o cardiovascolare (15).

Le modificazioni della posologia e della tipologia dei vari farmaci immunosoppressori nel corso degli anni vanno effettuate con estrema cautela e solo sulla base di precise indicazioni cliniche dettate da una accurata diagnosi, avallata se possibile dal riscontro morfologico bioptico renale. Inoltre va tenuta in considerazione la "storia" immunologica del paziente e la eventuale presenza di complicanze e co-morbidità.

Per quanto riguarda la progressiva riduzione del dosaggio degli immunosoppressori, in realtà nella pratica clinica si osserva che, nel lungo termine, nel paziente "stabilizzato" si tende a mantenere il livello ematico dei farmaci verso il limite inferiore del range suggerito. Dosaggi estremamente ridotti vanno monitorati con attenzione in quanto possono determinare la comparsa di rigetti sub-clinici spesso irreversibili.

Anche nell'ambito della medesima categoria, la sostituzione di un farmaco con un altro deve essere effettuata in base a precise motivazioni per effetti collaterali importanti. Spesso è sufficiente un adeguamento posologico o il frazionamento delle somministrazioni nella giornata per migliorare la sintomatologia,

In conclusione, non esiste a tutt'oggi lo schema terapeutico ottimale, ma esiste una strategia terapeutica, basata anche sull'esperienza del singolo Centro Trapianto, da adeguare alla storia naturale di ogni paziente con l'obiettivo di mantenere il rene trapiantato con una buona funzione il più a lungo possibile.

\section{Indirizzo degli Autori:}

Prof. Sergio Stefoni

U.O. di Nefrologia, Dialisi e Trapianto

Policlinico S. Orsola-Malpighi

Via Massarenti 9

40138 Bologna

sergio.stefoni@unibo.it

\section{Bibliografia}

1. Halloran PF. Immunosuppressive drugs for kidney tran splantation. N Engl J Med 2004; 351: 2715-29.

2. Hawksworth JS, Leeser D, Jindal RM, et al. New directions for induction immunosuppression strategy in solid organ transplantation. Am J Surg 2009; 197: 515-24.

3. Meier-Kriesche HU, Li S, Gruessner RW, et al. Immunosuppression: evolution in practice and trends, 1994-2004. Am J Transplant 2006; 6: 1111-31.

4. Serur D, Saal S, Wang J, et al. Deceased-donor kidney transplantation: improvement in long-term survival. Nephrol Dial Transplant 2011; 26: 317-24.

5. Cantarovich D, Vistoli F, Soulillou JP. Immunosuppression minimization in kidney transplantation. Front Biosci 2008; 13: 1413-32.

6. Pascual J, Zamora J, Galeano C, et al. Steroid avoidance or withdrawal for kidney transplant recipients. Cochrane Database Syst Rev 2009; 21; (1): CD005632.

7. Pascual J, Galeano C, Royuela A, Zamora J. A systematic review on steroid withdrawal between 3 and 6 months after kidney transplantation. Transplantation 2010; 90: 343-9.

8. Salvadori M, Scolari MP, Bertoni E, et al. Everolimus with very low-exposure cyclosporine a in de novo kidney transplantation: a multicenter, randomized, controlled trial. Transplantation 2009; 88: 1194-202.

9. Ekberg H, Bernasconi C, Tedesco-Silva H, et al. Calcineurin inhibitor minimization in the Symphony study: observational results 3 years after transplantation. Am J Transplant 2009; 9: 1876-85.

10. Lebranchu Y, Thierry A, Toupance O, et al. Efficacy on renal function of early conversion from cyclosporine to sirolimus 3 months after renal transplantation: concept study. Am J Transplant 2009; 9: 1115-23.

11. Pascual J. Everolimus in clinical practice-renal transplantation. Nephrol Dial Transplant 2006; 21: 18-23.

12. Grinyó JM, Cruzado JM. Mycophenolate mofetil and sirolimus combination in renal transplantation. Am J Transplant 2006; 6: 1991-9.

13. Flechner SM, Goldfarb D, Solez K, et al. Kidney transplantation with sirolimus and mycophenolate mofetil-based immunosuppression: 5-year results of a randomized prospective trial compared to calcineurin inhibitor drugs. Transplantation 2007; 83: 883-92.

14. Srinivas TR, Schold JD, Guerra G, et al. Mycophenolate mofetil/sirolimus compared to other common immunosuppressive regimens in kidney transplantation. Am J Transplant 2007; 7: 586-94.

15. Racusen LC, Regele H. The pathology of chronic allograft dysfunction. Kidney Int 2010; 78 (Suppl 119): S27-32. 